\title{
Elaboração e validação de instrumento de avaliação de tecnologia assistiva*
}

\section{Elaboration and validation of an assistive technology assessment questionnaire}

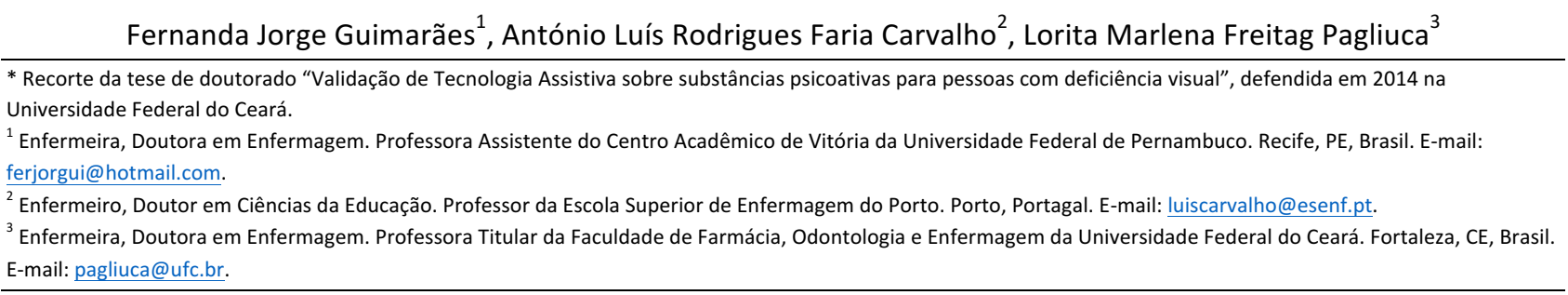

\section{RESUMO}

Tecnologias Assistivas consistem em recursos, métodos, estratégias que favorecem autonomia e inclusão de idosos e pessoas com deficiência, sendo escassos na literatura instrumentos que as avaliam. Estudo metodológico realizado com painel de especialistas e pessoas com deficiência visual, cujo objetivo foi elaborar e validar instrumento para avaliação de tecnologia assistiva educativa. Para se considerar um item do instrumento válido utilizou-se percentual de $80 \%$ de concordância, e foram calculadas a validade e a confiabilidade do instrumento. A Tecnologia Assistiva foi caracterizada em seis atributos: objetivos, acesso, clareza, estrutura e apresentação, relevância e eficácia, interatividade, para os quais foram elaborados 19 itens para a composição do instrumento. Destes, 11 obtiveram percentuais acima de 80\%, sete foram modificados e um excluído. $O$ alpha de Cronbach do instrumento foi de 0,822 , o que garante a validade e confiabilidade do instrumento para avaliar Tecnologia Assistiva de educação em saúde, e indica-se, portanto, o seu uso. Descritores: Educação Especial; Validade dos Testes; Estudos de Validação; Reprodutibilidade dos Testes; Equipamentos de Autoajuda.

\section{ABSTRACT}

Assistive Technologies consists of resources, methods, and strategies favoring autonomy and inclusion of elderly and people with disabilities, being scarce in the literature instruments assessing them. A methodology study conducted with a panel of specialists and people with visual impairment, aimed to elaborate and validate a questionnaire to assess educational assistive technology. To consider an item as valid, we used $80 \%$ as agreement percentage, and validity and reliability of the questionnaire were calculated. Assistive Technology was characterized in six attributes: objectives, access, clarity, structure and presentation, relevance and efficacy, interactivity, and 19 items were elaborated to compose the questionnaire. From those, 11 obtained percentages higher than $80 \%$, seven were modified and one was excluded. The instrument Cronbach's alpha was 0,822 , guaranteeing validity and reliability of the tool to assess health education Assistive Technology, and therefore, its use is indicated.

Descriptors: Education, Special; Validity of Tests; Validation Studies; Reproducibility of Results; Self-Help Devices. 


\section{INTRODUÇÃO}

Diversos materiais, métodos, estratégias podem ser usados como Tecnologia Assistiva (TA), que auxiliam pessoas idosas e pessoas com deficiência em suas atividades diárias. Tecnologia Assistiva pode ser conceituada como "área do conhecimento, de característica interdisciplinar, que engloba produtos, recursos, metodologias, estratégias, práticas e serviços que objetivam promover a funcionalidade e participação de pessoas com deficiência, visando sua autonomia, independência, qualidade de vida e inclusão social” ${ }^{(1)}$. Os principais objetivos da TA são promover acessibilidade, qualidade de vida e inclusão(2).

O uso da TA possibilita o envolvimento de vários profissionais, como terapeutas ocupacionais, fonoaudiólogos, fisioterapeutas, psicólogos, enfermeiros, assistentes sociais, oftalmologistas, especialistas em audição, protéticos $^{(3)}$. São consideradas Tecnologias Assistivas materiais e produtos para auxílio em tarefas como comer e vestir, recursos que permitem a comunicação, controle de aparelhos eletrônicos, sistemas de segurança, projetos para acessibilidade, órteses e próteses, adequação postural, cadeiras de roda, bases móveis, andadores, lupas e lentes, Braille para equipamentos com síntese de voz, grandes telas de impressão, telefones com teclado - teletipo (TTY), sistemas com alerta táctil-visual e recursos de acessibilidade ao computador (síntese de voz, teclados modificados, softwares especiais) ${ }^{(2)}$.

Estudo identificou como principais atributos de uma Tecnologia Assistiva: área do conhecimento interdisciplinar, recursos, serviços, métodos, estratégias, práticas, produtos, tecnologia, equipamentos ${ }^{(4)}$. Estes atributos são importantes, pois auxiliam na caracterização de uma Tecnologia Assistiva, sendo fundamental que essas sejam avaliadas.

O uso de instrumentos válidos e confiáveis é necessário para afirmar a adequação dessas tecnologias no atendimento da finalidade para a qual se propõem, no entanto após revisão em bases de dados, verificou-se que tais instrumentos são escassos na literatura. Dessa forma, observa-se uma lacuna na produção do conhecimento nesta área, pois uma TA adequada é importante para promover autonomia e qualidade de vida para idosos e pessoas com deficiência e, um instrumento validado contribui para uma efetiva avaliação da qualidade da mesma.

Dessa forma, identificou-se na literatura um instrumento que avalia a satisfação de usuários de Tecnologia Assistiva, denominado Quebec User Evaluation of Satisfaction with Assistive Technology (QUEST 2.0) ${ }^{(5)}$. Este instrumento pode ser aplicado como ferramenta clínica ou para pesquisa, e possui 12 itens, que são avaliados em escala tipo Likert com cinco pontos. Os itens avaliam peso, altura, fixação, segurança e serviço da tecnologia, o que permite utilizá-lo para avaliar diversas Tecnologias Assistivas. Entretanto, observou-se que o instrumento é mais adequado para avaliar Tecnologias Assistivas do tipo duras, como cadeiras, bases fixas, entre outras.

Por essa razão considera-se necessária a elaboração de um instrumento, que avalie TA educativa, como textos, vídeos, áudios, manuais, os quais são úteis para fornecer informação aos seus usuários sobre os cuidados com a saúde.

Ao observar o tipo de TA, os pesquisadores podem necessitar desenvolver medida para um construto particular, o que aperfeiçoa a operacionalidade do construto conforme o pesquisador o define ${ }^{(6)}$.

As Tecnologias Assistivas educativas são relevantes estratégias de informoterapia (information therapy). Nestas, devem-se considerar a acessibilidade e características intrínsecas dos suportes tecnológicos e da informação fornecida, quando utilizada como estratégia terapêutica de educação pelo profissional de enfermagem $^{(7)}$. Ao considerar esses aspectos é preciso garantir que a informação certa, chega à pessoa certa e na hora certa.

Ao verificar as necessidades identificadas para melhor qualificar o cuidado para pessoas que dependem 
das Tecnologias Assistivas, este estudo teve como objetivo elaborar e validar instrumento para avaliação de tecnologia assistiva educativa.

\section{MÉTODO}

Estudo metodológico, cujo enfoque é a construção e validação de instrumentos ${ }^{(8)}$. De acordo com o referencial adotado, a elaboração de instrumentos ocorre em três etapas, denominadas de polos, a saber: polo teórico, polo experimental e polo analítico. O polo teórico enfoca a questão da teoria que envolve o construto para o qual se quer desenvolver um instrumento de medida, como também a operacionalização do construto em itens. $O$ polo empírico define as etapas e técnicas de aplicação do instrumento piloto para a avaliação da qualidade psicométrica do instrumento. Finalmente, o polo analítico estabelece os procedimentos de análises estatísticas a serem efetuadas sobre os dados para conduzir a um instrumento válido, preciso e normatizado ${ }^{(8)}$.

O polo teórico é constituído por diversas etapas, e se inicia com a delimitação do sistema psicológico ou construto, que se pretende medir. Os sistemas psicológicos ou construtos não podem ser medidos, no entanto, podem ser medidas suas propriedades ou atributos. Dessa forma, segue-se a etapa de definição dos atributos do sistema psicológico ou construto, para os quais se quer construir o instrumento de medida. Para delimitar os atributos, sugere-se recorrer a experiência do pesquisador, ajuda da literatura e peritos na área ${ }^{(8)}$.

Em seguida, são elaboradas as definições constitutivas e operacionais para os atributos. As definições constitutivas são aquelas que comumente aparecem em dicionários e enciclopédias. Uma definição é operacional quando o construto é definido não mais em termos de conceitos, mas em termos de comportamentos físicos, que refletem a expressão desse construto. Por fim, é realizada a análise teórica por especialistas, que opinam sobre a pertinência dos itens do instrumento e, a análise semântica por grupos da população alvo, que confirmam a compreensão dos itens ${ }^{(8)}$.
No polo experimental ocorrem as etapas de planejamento da aplicação do instrumento, em que são definidas a amostra e as instruções de como aplicá-lo, e a coleta da informação. Por sua vez, no polo analítico há a análise dos dados ${ }^{(8)}$.

Neste estudo, realizou-se, primeiramente, a etapa do polo teórico. Assim, Tecnologia Assistiva educativa foi o construto selecionado. Em seguida, realizou-se revisão da literatura sobre Tecnologias Educativas e Tecnologias Assistivas educativas, que apontou como seus atributos: objetivos, acessibilidade, clareza, estrutura e apresentação, relevância e eficácia, interatividade ${ }^{(4,9-10)}$. Em seguida, apoiados na literatura, foram elaboradas as definições constitutivas e operacionais para cada atributo, como descrito a seguir.

Objetivos da TA são definidos como propósitos, metas ou fins a serem atingidos com sua utilização ${ }^{(9-10)}$. Acesso consiste no grau de facilidade de acesso à TA, se é inteligível e compreensível; como também, o direito de acessar a rede de informações, de eliminação de barreiras arquitetônicas, de disponibilidade de comunicação, de acesso físico, de equipamentos e programas adequados; de conteúdo e apresentação da informação em formatos alternativos $^{(11)}$.

Por sua vez, clareza do conteúdo indica se a informação apresentada pela TA é de fácil entendimento. O enunciado da informação é claro no sentido da transmissão do conteúdo ${ }^{(12)}$. Estrutura e apresentação do conteúdo da TA referem-se à forma de apresentar as informações e, inclui sua organização geral, estrutura, estratégia de apresentação, coerência e formatação ${ }^{(10)}$. Relevância e eficácia referem-se às características que avaliam o grau de significação do material educativo apresentado, à capacidade do material em causar impacto, motivação e/ou interesse, assim como ao grau de significação do material apresentado ${ }^{(9-10)}$.

Interatividade consiste no envolvimento do sujeito no processo educativo de uma maneira ativa e participante, podendo transformar algo que estava preestabelecido em situações didáticas, variadas e 
interessantes, ao ponto de provocar tal envolvimento. Diz respeito, também, a potencialidade de o usuário trilhar caminhos e acessar informações de acordo com o seu interesse e ritmo de trabalho ${ }^{(13)}$. A interatividade estimula os processos e escolhas dos sujeitos envolvidos na atividade educativa, o que fortalece o ensino e aprendizagem $^{(14)}$.

Elaboradas as definições constitutivas e operacionais para os atributos, desenvolveu-se a construção dos itens do Instrumento de Avaliação, que foram analisados por especialistas.

Participaram desta etapa seis especialistas, que possuíam experiência prática e produção científica relacionada aos temas tecnologia assistiva, elaboração de instrumentos ou pessoa com deficiência. Os especialistas foram identificados a partir de busca na Plataforma Lattes, contatados por correio eletrônico, e que por esta via responderam à pesquisa.

A avaliação dos itens devia valorar os critérios: clareza de linguagem, pertinência prática, relevância teórica e dimensão teórica. O critério de clareza de linguagem considera a linguagem utilizada nos itens, tendo em vista as características da população respondente. Pertinência prática considera se cada item foi elaborado de forma a avaliar o conceito de interesse em uma determinada população, analisa se de fato cada item possui importância para o instrumento. 0 critério relevância teórica, visa analisar se o item está relacionado com o construto e dimensão teórica, investiga a adequação de cada item à teoria estudada ${ }^{(8)}$. Cada critério foi avaliado por meio de escala tipo Likert com três pontos: 0 inadequado, 1 parcialmente adequado e 2 adequado.

Utilizou-se o percentual de concordância de $80 \%$ entre os especialistas como critério de decisão sobre a pertinência do item no instrumento. Após a análise das respostas dos especialistas, foram realizadas correções e obteve-se o instrumento, que foi submetido para avaliação por pessoas com deficiência. Esta última avaliação teve como objetivo verificar se membros da população alvo compreenderam os itens.

O polo experimental foi realizado com a aplicação do instrumento com pessoas com deficiência visual em associações ou institutos dos cegos nas cidades de Recife, Natal, João Pessoa, Fortaleza e Teresina. Um contato inicial com os diretores destas instituições foi feito para se apresentar o estudo. A partir daí, os diretores indicaram membros destas instituições, que auxiliaram a pesquisadora no contato com seus usuários. Ao entrar em contato com o usuário, inicialmente se apresentava os objetivos da investigação, e posteriormente realizava-se a leitura do Termo de Consentimento Livre e Esclarecido, solicitando ao participante sua assinatura. Após esses cuidados, a pesquisadora aplicava o instrumento.

Após o polo experimental, realizou-se o polo analítico, que consiste em analisar os dados coletados. Os dados foram inseridos em planilha Excel e analisados com uso do software Statistical Package for the Social Sciences (SPSS), versão 19. O nível de confiança adotado foi de $95 \%$.

Por fim, calculou-se a validade e fidedignidade do instrumento. A validade foi verificada pela análise fatorial. A análise em componentes principais foi seguida de rotação varimax, um método usado quando se pretende obter uma estrutura simples, uma vez que se trata de uma técnica que maximiza as saturações mais elevadas e reduz as saturações mais baixas.

Em relação à fidedignidade do instrumento, utilizouse o cálculo da consistência interna, especificamente o coeficiente Alpha de Cronbach.

O estudo foi aprovado pelo Comitê de Ética e Pesquisa da Universidade Federal de Pernambuco (CAAE 08796212.2.0000.5208).

\section{RESULTADOS}

O painel de especialistas foi constituído por seis profissionais de diferentes áreas do conhecimento, como Enfermagem, Pedagogia, Fisioterapia, Terapia Ocupacional e Ciências da Computação. No que se refere 
ao tempo de experiência de trabalho, este variou de seis a 38 anos, com a média de 20,33 anos. Todos os selecionados apresentaram produção científica nas áreas de desenvolvimento de tecnologia assistiva, pessoa com deficiência ou educação inclusiva. Um dos especialistas desenvolve pesquisa relacionada à elaboração de instrumentos. Quanto à titulação, dois possuem o título de mestre e os demais, título de doutor.

A partir da definição dos atributos de Tecnologia Assistiva descritos anteriormente, elaboraram-se 19 itens, que foram avaliados por painel de especialistas. $\mathrm{Na}$ Tabela 1 estão descritos os itens e o percentual de concordância obtido em cada critério avaliado.

Tabela 1: Distribuição dos itens de acordo com o percentual de concordância dos especialistas. Fortaleza, CE, Brasil, 2014.

\begin{tabular}{|c|c|c|c|c|c|}
\hline Atributos & Itens & $\begin{array}{c}\text { Clareza de } \\
\text { linguagem } \\
\text { (\%) }\end{array}$ & $\begin{array}{l}\text { Pertinência } \\
\text { prática (\%) }\end{array}$ & $\begin{array}{l}\text { Relevância } \\
\text { teórica (\%) }\end{array}$ & $\begin{array}{l}\text { Dimensão } \\
\text { teórica (\%) }\end{array}$ \\
\hline \multirow{5}{*}{ Objetivos } & Relaciona o conteúdo abordado & & & & \\
\hline & $\begin{array}{c}\text { na Tecnologia Assistiva no seu dia } \\
\text { a dia }\end{array}$ & 83 & 100 & 83 & 100 \\
\hline & $\begin{array}{l}\text { Esclarece dúvidas sobre o } \\
\text { conteúdo abordado }\end{array}$ & 100 & 83 & 83 & 83 \\
\hline & $\begin{array}{l}\text { Estimula a aprendizagem sobre o } \\
\text { conteúdo abordado }\end{array}$ & 83 & 100 & 100 & 100 \\
\hline & $\begin{array}{l}\text { Permite-Ihe buscar informações } \\
\text { sem dificuldades }\end{array}$ & 100 & 100 & 100 & 100 \\
\hline \multirow[t]{2}{*}{ Acesso } & $\begin{array}{c}\text { Disponibiliza os recursos } \\
\text { adequados e necessários para seu } \\
\text { manuseio }\end{array}$ & 100 & 100 & 100 & 100 \\
\hline & Apresenta informação concisa & 50 & 83 & 83 & 83 \\
\hline \multirow[b]{2}{*}{ Clareza } & $\begin{array}{l}\text { Conteúdo da informação está } \\
\text { adequado à realidade do usuário }\end{array}$ & 66 & 100 & 100 & 100 \\
\hline & $\begin{array}{l}\text { Descreve os conceitos } \\
\text { apresentados }\end{array}$ & 66 & 100 & 100 & 100 \\
\hline \multirow{3}{*}{$\begin{array}{c}\text { Estrutura e } \\
\text { apresentação }\end{array}$} & Apresenta conteúdo estruturado & 66 & 100 & 100 & 100 \\
\hline & Apresenta conteúdo organizado & 66 & 100 & 100 & 100 \\
\hline & $\begin{array}{l}\text { Apresenta aspectos relevantes do } \\
\text { conteúdo abordado }\end{array}$ & 66 & 100 & 100 & 100 \\
\hline \multirow{4}{*}{$\begin{array}{l}\text { Relevância e } \\
\text { eficácia }\end{array}$} & $\begin{array}{l}\text { Permite-lhe refletir sobre o } \\
\text { conteúdo apresentado pela TA }\end{array}$ & 83 & 100 & 100 & 100 \\
\hline & $\begin{array}{l}\text { Desperta interesse para utilizar a } \\
\text { Tecnologia Assistiva }\end{array}$ & 83 & 100 & 100 & 100 \\
\hline & $\begin{array}{l}\text { Estimula mudança de } \\
\text { comportamento }\end{array}$ & 83 & 100 & 83 & 83 \\
\hline & $\begin{array}{l}\text { Reproduz o conteúdo abordado } \\
\text { em diferentes contextos }\end{array}$ & 100 & 100 & 100 & 100 \\
\hline \multirow{4}{*}{ Interatividade } & $\begin{array}{c}\text { Oferece interação com a } \\
\text { Tecnologia Assistiva }\end{array}$ & 50 & 100 & 100 & 100 \\
\hline & $\begin{array}{c}\text { Possibilita navegar sem } \\
\text { dificuldades pelos links } \\
\text { apresentados pela TA }\end{array}$ & 83 & 100 & 100 & 100 \\
\hline & $\begin{array}{l}\text { Eficiência na operação dos tópicos } \\
\text { que foram colocados a disposição } \\
\text { para acesso da TA }\end{array}$ & 66 & 100 & 100 & 100 \\
\hline & $\begin{array}{l}\text { Fornece autonomia ao usuário em } \\
\text { relação à operação da TA }\end{array}$ & 83 & 100 & 100 & 100 \\
\hline
\end{tabular}


Dos itens avaliados, oito apresentaram percentual de concordância inferior a $80 \%$ no critério clareza de linguagem. Destes, sete itens foram reformulados e um foi excluído, conforme as sugestões dos especialistas. Os itens discordantes no critério clareza de linguagem avaliavam as áreas: acesso, clareza do conteúdo abordado na TA e estrutura e apresentação.

Quanto aos critérios de pertinência prática, relevância teórica e dimensão teórica, os itens alcançaram um percentual de concordância entre os especialistas igual ou superior a $80 \%$. Desta forma, todos os itens foram considerados representativos para avaliar
Tecnologias Assistivas, educativas, como textos educativos, vídeos, manuais, entre outros.

Os itens foram distribuídos de acordo com os atributos identificados para Tecnologia Assistiva educativa. Quatro itens julgam os objetivos da tecnologia, dois avaliam acesso a TA, três clareza, dois avaliam estrutura e apresentação do conteúdo da TA, quatro relevância e eficácia e três interatividade. Concluída a avaliação por painel de especialistas, os itens foram inseridos no instrumento, constituído por 18 itens, como apresentado no Quadro 1.

Quadro 1: Distribuição dos itens do instrumento. Fortaleza, CE, Brasil, 2014.

\begin{tabular}{|c|c|c|c|c|c|}
\hline Atributos & & Item & 0 & 1 & 2 \\
\hline \multirow{4}{*}{ Objetivos } & 1 & Relaciona o conteúdo abordado no seu dia a dia & & & \\
\hline & 2 & Esclarece as dúvidas sobre o conteúdo abordado & & & \\
\hline & 3 & Estimula aprendizagem sobre o conteúdo abordado & & & \\
\hline & 4 & Estimula aprendizagem de novos conceitos ou fatos & & & \\
\hline \multirow{2}{*}{ Acesso } & 5 & Permite-Ihe buscar informações sem dificuldades & & & \\
\hline & 6 & Disponibiliza recursos adequados e necessários para sua utilização & & & \\
\hline \multirow{3}{*}{ Clareza } & 7 & Apresenta informações necessárias para melhor compreensão do conteúdo & & & \\
\hline & 8 & Conteúdo da informação está adequado às suas necessidades & & & \\
\hline & 9 & Apresenta informações de modo simples & & & \\
\hline \multirow{2}{*}{ Estrutura e apresentação } & 10 & Apresenta conteúdo de forma organizada & & & \\
\hline & 11 & Possui estratégia de apresentação atrativa & & & \\
\hline \multirow{4}{*}{ Relevância e eficácia } & 12 & Permite-lhe refletir sobre o conteúdo apresentado & & & \\
\hline & 13 & Desperta seu interesse para utilizá-la & & & \\
\hline & 14 & Estimula mudança de comportamento em você & & & \\
\hline & 15 & Reproduz o conteúdo abordado em diferentes contextos & & & \\
\hline \multirow{3}{*}{ Interatividade } & 16 & Oferece interação, envolvimento ativo no processo educativo & & & \\
\hline & 17 & Possibilita navegar sem dificuldades pelos links apresentados & & & \\
\hline & 18 & Fornece autonomia ao usuário em relação à sua operação & & & \\
\hline
\end{tabular}

As instruções, que acompanham o instrumento, informam que o participante deverá atribuir nota 0 , quando julgar o item inadequado, 1 parcialmente adequado ou 2 quando considerar o item adequado. Para se considerar o questionário válido é importante que todos os itens sejam respondidos.

Concluída a análise por especialistas, o instrumento foi submetido à análise semântica pelos sujeitos da população alvo e todos os itens foram considerados claros e compreensíveis pelos participantes da população
- alvo para o qual o instrumento foi elaborado e não foi necessário modificá-los.

No que diz respeito ao polo experimental, participaram 140 pessoas com deficiência visual, dos quais $65,7 \%$ eram do gênero masculino, com idade média de 37,1 anos e média de escolaridade de 10,1 anos. No tocante ao tipo de deficiência visual, $84,3 \%$ dos participantes possuem cegueira e $15,7 \%$ baixa visão.

No que diz respeito a validade, inicialmente, verificou-se a análise da matriz de correlações entre os itens e o total da escala, o que permitiu identificar que 
foram obtidos índices altamente significativos $(p<0,05)$, à exceção dos itens 1, 2, 7 e 10. Os demais itens apresentaram correlações significativas entre si e com o total do instrumento. A análise fatorial, atendendo ao critério de decisão de Guttman-Kaiser, revelou a existência de quatro atributos, como observado na Tabela 2.

Tabela 2: Distribuição dos itens do instrumento de acordo com os atributos. Fortaleza, CE, Brasil, 2014.

\begin{tabular}{ccccc}
\hline Itens & \multicolumn{3}{c}{ Atributos } & \multicolumn{2}{c}{$\mathbf{h}^{\mathbf{2}}$} \\
\cline { 2 - 4 } 8 & $\mathbf{1}$ & $\mathbf{2}$ & $\mathbf{3}$ & $\mathbf{4}$ \\
16 & 0,704 & & 0,586 \\
17 & 0,495 & & 0,352 \\
18 & 0,769 & & 0,649 \\
3 & 0,593 & & 0,461 \\
4 & & 0,802 & & 0,675 \\
5 & & 0,756 & & 0,663 \\
11 & & 0,599 & & 0,552 \\
6 & & 0,510 & & 0,483 \\
13 & & & 0,547 & 0,642 \\
14 & & 0,634 & 0,643 \\
15 & & 0,800 & 0,684 \\
9 & & 0,479 & 0,508 \\
12 & & & 0,442 \\
\% Variância & & & 0,690 \\
\end{tabular}

Testes: Rotação varimax. Análise fatorial.

Após essa análise retirou-se, portanto, os quatro itens do instrumento e por fim, calculou-se o alpha de Cronbach, que foi de 0,822 .

Finalizada a análise das medidas psicométricas permaneceram quatro atributos com 14 itens e, o instrumento pode ser considerado válido e confiável. A versão final do Instrumento de Avaliação de Tecnologia Assistiva encontra-se em seguida, precedido das orientações de preenchimento.

\section{Instrumento de Avaliação de Tecnologia Assistiva}

Este instrumento tem por objetivo registrar a sua avaliação em relação à Tecnologia Assistiva (TA). Para cada atributo, você deverá atribuir nota de 0 a 2, como desejar, de acordo com a legenda abaixo:

- Inadequado: a tecnologia assistiva não atende a definição do item.

- Parcialmente adequado: a tecnologia atende parcialmente a definição do item.
- Adequado: a tecnologia atende a definição do item.

Caso seja do seu interesse, você poderá comentar, criticar ou sugerir os aspectos que considerou como positivos ou negativos na TA. 
Quadro 2: Parte integrante do Instrumento de Avaliação de Tecnologia Assistiva. Fortaleza, CE, Brasil, 2014.

\begin{tabular}{|c|c|c|c|c|c|}
\hline Atributos & & Item & 0 & 1 & 2 \\
\hline \multirow{4}{*}{1 Interatividade } & 1 & O conteúdo está adequado às suas necessidades & & & \\
\hline & 2 & Oferece interação e envolvimento no processo educativo & & & \\
\hline & 3 & Possibilita acessar os tópicos apresentados & & & \\
\hline & 4 & Fornece autonomia ao usuário em relação à sua operação & & & \\
\hline \multirow{4}{*}{2 Objetivos } & 5 & Estimula aprendizagem sobre conteúdo abordado & & & \\
\hline & 6 & Estimula aprendizagem de novos conceitos & & & \\
\hline & 7 & Permite buscar informações sem dificuldades & & & \\
\hline & 8 & Possui estratégia de apresentação atrativa & & & \\
\hline \multirow{4}{*}{3 Relevância e eficácia } & 9 & Disponibiliza recursos adequados para utilização & & & \\
\hline & 10 & Desperta interesse para utilizá-la & & & \\
\hline & 11 & Estimula mudança de comportamento & & & \\
\hline & 12 & Reproduz o conteúdo abordado em diferentes contextos & & & \\
\hline \multirow{2}{*}{4 Clareza } & 13 & Apresenta informações de modo simples & & & \\
\hline & 14 & Permite refletir sobre o conteúdo apresentado & & & \\
\hline
\end{tabular}

\section{DISCUSSÃO}

No exercício de sua prática, os profissionais têm como foco promover maior autonomia às pessoas com deficiência. Com a disponibilidade do instrumento de avaliação de Tecnologia Assistiva educativa espera-se que os profissionais e usuários possam avaliar sua adequação aos objetivos propostos, especialmente, quando as utiliza como ferramenta de informação terapêutica.

A informação terapêutica baseia-se em evidências para um determinado paciente, cuidador, ou consumidor no momento certo para ajudá-los a tomar uma decisão de saúde específica ou tomar uma ação de autogestão eficaz do regime terapêutico ${ }^{(15)}$. A prescrição da informação certa, no momento certo e na dose adequada faz parte do processo de cuidados, abre portas a uma nova forma de pensar a Enfermagem, surgindo aí uma nova oportunidade de prestação de cuidados de saúde.

Estudo realizado com pacientes com doença pulmonar obstrutiva crônica identificou que os recursos educacionais podem adotar diferentes suportes tecnológicos, desde que adequados às necessidades informacionais dos pacientes ${ }^{(7)}$. Este resultado assemelha-se ao presente estudo, pois por meio de um instrumento adequado é possível avaliar a capacidade informacional da Tecnologia Assistiva educativa, que tem como objetivo informar e promover a autonomia do usuário. A informação com qualidades terapêuticas para a pessoa com deficiência deve ser focada na decisão, baseada na evidência, revista por especialistas, atualizada e compreensível para a pessoa para quem foi prescrita.

De igual forma, os achados deste estudo, corrobora estudo realizado com cegos sobre doenças sexualmente transmissíveis, que apontou para a necessidade de iniciativas acessíveis de promoção da saúde, como a informação em saúde, o diálogo, materiais táteis, a esta população exposta a diversos fatores de risco ${ }^{(16)}$.

Os resultados apresentados neste estudo assemelham-se a outro que desenvolveu escala de satisfação para uso de Tecnologia Assistiva, o qual realizou a validação de conteúdo com painel de especialistas, que avaliaram a versão preliminar do instrumento. Neste nível, os resultados revelaram que o instrumento inclui todos os aspectos importantes de satisfação de Tecnologia Assistiva, como também se verificou a necessidade de alterações na redação dos itens e procedimentos para garantir a ótima validade de conteúdo $^{(5)}$. A validação de conteúdo por especialistas constitui uma das etapas para a elaboração de instrumentos ${ }^{(17)}$. Essa etapa se refere à fase em que os itens do instrumento representam adequadamente o construto que se pretende avaliar ${ }^{(18)}$.

Nesta etapa, o painel de especialistas fornece um retorno construtivo sobre a qualidade da medida recentemente construída e critérios objetivos para avaliar 
cada item. Além disso, oferecem sugestões concretas para melhorar a medida ${ }^{(6)}$. Possibilita aprimorar e legitimar um novo instrumento de medida que está sendo proposto, o que é relevante para criar instrumentos válidos, confiáveis no atual contexto de saúde ${ }^{(19)}$. Portanto, há evidências da relevância de estudos de validação de conteúdo, embora apresente como principal limitação a subjetividade da análise dos especialistas, como também há divergências entre o número ideal de especialistas.

\section{CONCLUSÃO}

O processo de validação do instrumento de avaliação de tecnologia assistiva educativa apoiou-se em revisão de literatura, que identificou os atributos objetivos, acesso, clareza, estrutura e apresentação, relevância e eficácia e, interatividade com 19 itens. Após avaliação por especialistas, o instrumento manteve os atributos, e foram reformulados sete itens e um foi excluído. O instrumento resultante desta etapa foi submetido ao público alvo, pessoas com deficiência visual.

\section{REFERÊNCIAS}

1. Coordenadoria Nacional de Promoção dos Direitos da Pessoa com Deficiência [internet]. Ata VII reunião do Comitê de Ajudas Técnicas. [cited 2011 mar 10] Available from: http://portal.mj.gov.br/corde. 2. Secretaria de atenção à saúde; Ministério da saúde. Política Nacional de Saúde da Pessoa com Deficiência. Brasília: Ministério da Saúde; 2010.

3. Pelossi MB, Nunes LROP. Caracterização dos professores itinerantes, suas ações na área de tecnologia assistiva e seu papel como agente de inclusão escolar. Rev. bras. educ. espec. [Internet]. 2009 [cited 2012 jan 14];15(1):141-54. Available from:

http://www.scielo.br/scielo.php?pid=S141365382009000100010\&script=sci_abstract\&tlng=pt.

4. Guimarães FJ, Pagliuca LMF. Assistive technology: an analysis of the concept. Rev. enferm. UFPE on line. [Internet]. 2012 [cited 2013 feb 27];6(11):915-23. Available from:

http://www.revista.ufpe.br/revistaenfermagem/index.php/revista/arti cle/viewArticle/2902

5. Demers L, Weiss-lambrou R, Ska B. The Quebec User Evaluation of Satisfaction with Assistive Technology (QUEST 2.0): an overview and recent progress. Technol. disabil [Internet]. 2002 [cited 2013 feb 27];14:101-5. Available from: https://enablemob.wustl.edu/OT572D01/RequiredArticles/QUEST\%20article.pdf.

6. Rubio DM, Berg-Weger M, Tebb SS, Lee ES, Rauch S. Objectifying content validity: conducting a content validity study in social work
A análise estatística com alpha de Cronbach de 0,822 consolidou o Instrumento de Avaliação de Tecnologia Assistiva com quatro atributos: interatividade, objetivos, relevância e eficácia e, clareza, distribuídos em 14 itens.

A intenção na realização do estudo era contribuir com a elaboração de Tecnologias Assistivas educativas, que possam ser avaliadas por instrumentos confiáveis. De uma maneira geral, este estudo de validação forneceu melhor direção para a revisão da medida investigada.

$\mathrm{O}$ instrumento aqui proposto foi considerado como prático para avaliar Tecnologias Assistivas educativas destinadas a pessoas com deficiência ou pessoas idosas que as usam.

Os resultados apresentados contribuem para o incremento do conhecimento na temática, no entanto, o instrumento proposto alcança apenas a avaliação de Tecnologias Assistivas educativas, não sendo recomendável sua aplicação para outros tipos de TA.

O instrumento validado é adequado para ser utilizado por pessoas com deficiência para avaliarem objetos educativos acessíveis, o que o torna uma inovação no contexto dos cuidados a esta clientela.

research. J. soc. social. work. res. [Internet] 2003 [cited 2013 feb 27];27(2):94-104. Available from: http://swr.oxfordjournals.org/content/27/2/94.abstract. 7. Padilha JMSC, Sousa PAF, Pereira FMS. Análise do uso de suportes tecnológicos e conteúdos informacionais pelos pacientes com doença pulmonar obstrutiva crônica. Acta Paul. Enferm. [Internet] 2012 [cited 2013 jul 24];25(especial):60-6. Available from:

http://www.scielo.br/pdf/ape/v25nspe1/pt_10.pdf.

8. Pasquali L. Instrumentação psicológica: fundamentos e práticas. Porto Alegre: Artmed; 2010.

9. Nascimento MHM, Teixeira E. Tecnologia educacional para famílias cangurus em terapia intensiva neonatal: travessias entre textos e imagens. In: Nietsch EA, Teixeira E, Medeiros HP. Tecnologias cuidativo- educacionais: uma possibilidade para o empoderamento do(a) enfermeiro(a)? Porto Alegre: Moriá; 2014. p.197-213.

10. Oliveira MS, Fernandes AFC, Sawada NO. Manual educativo para o autocuidado da mulher mastectomizada: um estudo de validação. Texto Contexto enferm. [Internet] 2008 [cited 2013 sep 19];17(1):11523. Available from: http://www.scielo.br/scielo.php?script=sci_arttext\&pid=S010407072008000100013.

11. Acesso Brasil. O que é acessibilidade? [Internet]. Rio de Janeiro [cited 2013 jan 30] Available from:

http://www.acessobrasil.org.br/index.php?itemid=45. 
12. Vilarinho S. Clareza de um texto. [Internet]. [cited 2013 mar 12] Available from: http://www.brasilescola.com/redacao/a-clareza-umtexto.htm.

13. Braga AB, Maubrigades V. Avaliação de softwares educativos do departamento nacional SENAC. Rio de Janeiro: SENAC/ DN/ DIPLAN; 2005.

14. Rangel ML, Barbosa AO, Riccio NCR, Souza JS. Redes de aprendizagem colaborativas: contribuição da educação a distância no processo de qualificação de gestores do Sistema Único de Saúde - SUS. Interface [Internet]. 2012 [cited 2013 mar 07];16(41). Available from: http://www.scielo.br/scielo.php?script=sci_arttext\&pid=S141432832012000200019\&lng=en\&nrm=iso.

15. Mettler M, Kemper DW. Information Therapy: the strategic role of prescribed information in disease self-management. Int. j. rheum. Dis [Internet]. 2005 [cited 2013 mar 07];8:69-76. Available from: http://www.ncbi.nlm.nih.gov/pubmed/17095835.

16. Cezario KG, Mariano MR, Pagliuca LMF. Comparando o comportamento sexual de cegos e cegas diante das DSTs. Rev. Eletr. Enf. [Internet]. 2008 [cited $2013 \mathrm{dec} 13$ ];10(3):686-94. Available from: http://www.fen.ufg.br/revista/v10/n3/v10n3a14.htm.

17. Alexandre NMC, Coluci MZO. Content validity in the development and adaptation processes of measurement instruments. Cinc. saúde coletiva. [Internet] 2011 [cited $2013 \mathrm{dec}$ 10];16(7):3061-8. Available from: http://www.scielo.br/scielo.php?pid=S1413$81232011000800006 \&$ script=sci_arttext.

18. Kreis MK, Cooke DJ, Michie C, Hoff HA, Logan C. The compreensive assessment of psychophatic personality (CAPP): content validation using prototypical analysis. Personal ment health. [Internet] 2013 [cited 2013 may 10];26(3):402-13. Available from: http://www.ncbi.nlm.nih.gov/pubmed/22686228.

19. Amendola F, Alvarenga MRM, Gaspar JC, Yamashita $\mathrm{CH}$, Oliveira MAC. Validade aparente de um índice de vulnerabilidade das famílias a incapacidade e dependência. Rev. esc. enferm. USP. [Internet] 2011 [cited 2013 may 10];45(especial 2):1736-421. Available from: http://www.scielo.br/scielo.php?script=sci_arttext\&pid=S008062342011000800017.

Recebido: 09/03/2014.

Aceito: 06/03/2015.

Publicado: 30/06/2015. 